\title{
Plasmodium falciparum malaria resistance to chloroquine in five communities in Southern Nigeria
}

\author{
Patrick O Erah ${ }^{*}$, Gertrude Arienmughare and Augustine O Okhamafe \\ Pharmacotherapy Group, Faculty of Pharmacy, University of Benin, Benin City, Nigeria.
}

Accepted 29 September 2003

\begin{abstract}
Chloroquine is still a first-line antimalarial drug in uncomplicated falciparum malaria. Increasing resistance to chloroquine has been reported in many parts of Nigeria. Clinical and parasitological responses and classes of resistance to chloroquine in falciparum malaria in five communities in Delta region, southern Nigeria were assessed. Chloroquine was administered to 218 patients with uncomplicated $P$. falciparum malaria. The levels of parasitemia, clinical response and classes of resistance were monitored for 7 days. High levels of therapeutic failures of chloroquine in $P$. falciparum malaria were recorded in the region. The frequencies of clinical and parasitological failure of chloroquine were $\mathbf{2 5 . 7 \%}$ and $\mathbf{5 5 \%}$, respectively. These frequencies were significantly lower in children below 5 years than older people. R2 and R3 resistance occurred in $37.2 \%$ and $17.4 \%$ of the patients, respectively. The therapeutic failure of chloroquine was not gender dependent. We conclude that chloroquine is still effective in the treatment of uncomplicated $P$. falciparum malaria in some communities in Delta region of Nigeria. However, resistance to chloroquine is likely. These results may be used as an important indicator of the significant level of therapeutic failure of uncomplicated $P$. falciparum malaria to chloroquine in Nigeria.
\end{abstract}

Keywords: Chloroquine, falciparum malaria, resistance, clinical failure, parasitological failure.

\section{INTRODUCTION}

Malaria is the second leading health problem (the first being HIVIAIDS) in sub-Saharan Africa accounting for over 1 million deaths yearly in the region (Rathod et al., 1997). These deaths are primarily among children under 5 years of age and pregnant women (Phillips, 2001). The disease can increase the risk of major problems including anaemia, premature births, still-births, abortion and low birth weight. Health and socio-economic problems caused by malaria in sub-Saharan Africa are enormous, and the development and spread of malaria parasite resistance to drugs have led to global recognition and commitment to public health problems of malaria.

*Corresponding author; E-mail: erah@uniben.edu, Tel: +234 802336 0318; 805526 3622, Fax: +234 52602257.
Malaria in Africa is mainly due to a blood parasite, Plasmodium falciparum. Chloroquine, which was introduced in the 1940s, and for many decades served as a cheap and reliable drug, is becoming ineffective against P. falciparum in most tropical areas (Rathod et al., 1997; Warhurst, 2001). Resistance to chloroquine developed in Southeast Asia and South America at the end of the 1950s and in Africa by the late 1970s (Warhurst, 2001). Since the emergence of chloroquine resistant strains of $P$. falciparum, the rate of resistance has been increasing and limiting adequate treatment of malaria (Peters, 1998; Neequaye et al., 1986). Despite the growing resistance of $P$. falciparum, chloroquine remains the first line antimalarial drug in Nigeria and many other African countries mainly on account of cost and effectiveness in uncomplicated cases of malaria. Resistance to chloroquine by $P$. falciparum has prompted many studies 
within the last decade in different parts of Nigeria (Molta, 1995; Umotong et al., 1991; Salako et al., 1990; Sowunmi et al., 1990; Sowunmi and Salako, 1992). In the study carried out in northeastern Nigeria, for example, most strains of $P$. falciparum were found to be fully sensitive to chloroquine (Molta, 1995). However, strains with reduced sensitivity and resistant strains have emerged with parasitological failure increasing from $18.7 \%$ in 1988 to $24.5 \%$ in 1995 (Molta, 1995). Consistently high resistance to chloroquine by malaria parasite has also been reported in southeastern Nigeria (Umotong et al., 1991).

The goal of chemotherapy in malaria therapy is often to effect a clinical cure or parasitological clearance or to limit the development of drug resistance. Resistance to chloroquine is often manifested in subtle ways which are only apparent when the treatment is accompanied by detailed follow-up. Accurate and effective surveillance systems for monitoring antimalarial drug efficacy have been recognized as an essential basis for decisions on the use of drugs (Malaria Foundation International, 1998). Surveillance is often carried out at different levels of sophistication, from basic drug efficacy tests through to more detailed in vitro characterization of drug resistant parasite strains and clinical information. The World Health Organization has recognized that malaria treatment failure rate of $5 \%$ to $14 \%$ signals an alert phase in malaria monitoring while failure rates of $15 \%$ to $24 \%$ signals the need for action (WHO, 2002a). Among other factors, reports of chloroquine resistant $P$. falciparum treatment failure rate of up to $53.6 \%$ in southeastern Nigeria (Umotong et al., 1991) and up to $37 \%$ in southwestern part of Nigeria (Salako et al., 1990; Sowunmi et al., 1990) with a drop to $15 \%$ in 1992 (Sowunmi and Salako, 1992) justifies the need for continuous monitoring of resistance to chloroquine.

The main purpose of this study was to ascertain the therapeutic efficacy of treatment of uncomplicated $P$. falciparum malaria with chloroquine in the Delta region of Nigeria. Specifically, the parasitological and clinical responses of $P$. falciparum to chloroquine and the classes of resistance are evaluated. The data obtained from this study would provide useful information for future management of uncomplicated $P$. falciparum malaria in the region.

\section{METHOD}

\section{Study area}

This study was carried out in a government-owned Central Hospital, Warri which is a 300 -bed tertiary health care facility. It involved patients drawn from five communities in Delta State, Southern Nigeria namely, Agbarho, Aladja, Effurun, Ekpan and Enerhen with a population of over 1 million inhabitants. These communities are located in malaria endemic oil rich area of Delta State and were selected because of their proximity to the study site. Apart from Agharho (a village whose inhabitants are mainly farmers), the communities are urban communities whose inhabitants are mainly petty traders, civil servants and workers in the oil industry. They are served by the Central Hospital, Warri and Shell Petroleum Hospital, Warri as well as private clinics, herbal homes, pharmaceutical stores and patent medicine stores. Like other parts of southern Nigeria, the climate is equatorial, with 2 seasons: rainy season from May to October and dry season from November to April.

\section{Patients and study protocol}

After ethical approval from the Delta State Ministry of Health, Asaba, 500 males and females patients (age: 6 months and above) with history of fever (axillary temperature $\geq 37.5^{\circ} \mathrm{C}$ ) in the last $48 \mathrm{~h}$ before attending the hospital (Central Hospital, Warri) were clinically examined and screened for malaria parasites using both clinical examination and laboratory data. Of the 500 patients, 218 patients (age range, 6 months to 48 years) met the criteria for inclusion in the data analysed. These patients were selected from the 5 communities using simple random sampling as earlier described (WHO, 1992). The sample was evenly distributed among the communities selected and the sample size was satisfactory in respect of the calculated sample size of patients based on the population of the communities at $95 \%$ confidence interval, $5 \%$ precision, and expected proportion of treatment failure of $5 \%-14 \%$ (WHO, 2002a).

The criteria for inclusion in the study included the presence of $P$. falciparum malaria as determined by clinical examination and laboratory data (parasite count; 2,000 to 200,000 per $\mu$ l blood) and informed consent. Other criteria for inclusion were: (1) ability to be followed up for 7 days, (2) presence of febrile conditions caused by $P$. falciparum malaria, (3) absence of general danger signs or signs of severe and complicated $P$. falciparum malaria, and (4) the fact that the patient has not taken any antimalarial drug within 2 week period preceding the study. However, patients with clinical symptoms compatible with severe or complicated malaria or with any other symptoms or signs of non-malarial etiology were excluded from the study and referred to appropriate health services. Also excluded were patients with repeated vomiting, diarrhoea, malnutrition, pregnancy, difficulty in complying with drug treatment, and inability to comply with the stipulated follow-up visits on days 3 and 7.

The 218 patients (113 males and 105 females including 78 children below 5 years) were routinely treated with chloroquine orally: adults were treated with $600 \mathrm{mg}$ chloroquine base stat and $24 \mathrm{~h}$ later followed by $300 \mathrm{mg}$ next day; children received $10 \mathrm{mg} / \mathrm{kg}$ stat and 24 
Table 1. Age and sex distribution of patients with $P$. falciparum malaria treated with chloroquine.

\begin{tabular}{|l|l|l|l|}
\hline Age & Male $(\%)^{\text {a }}$ & ${\text { Female }(\%)^{\text {a }}}$ & Total (\%) \\
\hline 6 months to $<5$ years & $40(18.3)$ & $38(17.4)$ & $78(35.8)$ \\
$5-48$ years & $73(33.5)$ & $67(30.7)$ & $130(64.2)$ \\
Total & $113(51.8)$ & $105(48.2)$ & $218(100)$ \\
\hline
\end{tabular}

${ }^{a} \chi^{2}=0.015, d f=1, p=0.903$

$\mathrm{h}$ later, then $5 \mathrm{mg} / \mathrm{kg}$ next day, as appropriate. Each patient was also given appropriate doses of paracetamol and multivitamin orally. The qualities of drugs administered were evaluated and found to have met the British Pharmacopoeia (BP, 1998) standard before they were used for this study. All patients were treated as outpatients and were appropriately followed up for 7 days with clinical and laboratory examinations at $3 \mathrm{~h}$, day 3 and day 7 following the start of treatment with chloroquine. Blood samples were also obtained from the patients during the follow-up days and malaria parasite levels determined using standard methods (Ejov et al., 1999; Hombhanje, 1997).

\section{Clinical and parasitological responses to chloroquine}

Clinical failure was considered to have occurred if a patient had fever or history of fever (axillary temperature $\geq 37.5^{\circ} \mathrm{C}$ ) by day 7 with parasitemia detected in blood or assessed to have been ill due to no other cause than malaria. Parasitological failure was considered to have occurred if parasite count in blood was $\geq 25 \%$ of count on day 0 by day 7 .

\section{Level of resistance to chloroquine}

We adopted the 7-day WHO in vivo test protocol in determining the class of resistance to chloroquine (Bruce, 1986). Patients with marked reduction of parasitemia (parasite count reduced by more than $75 \%$ ) at $48 \mathrm{~h}$ but failed to clear parasites by day 7 were considered to be R2 resistant to chloroquine. Patients whose parasitemia did not fall by more than $75 \%$ within $48 \mathrm{~h}$ or occasionally increased by day 7 were considered to be R3 resistant to chloroquine. R1 resistance (clearance of asexual parasitemia on or after day 7 followed by recrudescence) was not determined because the patients were only monitored for 7 days and it is not possible to determine recrudescence in areas of intense malaria transmission as in the communities studied.

\section{Statistical Analysis of data}

The data obtained were categorized based on sex and age and reported as frequencies. Data were compared with Chi square test or Fisher's Exact test, using a computer software, Instat (GraphPad Inc., USA). Probability ( $p$ ) was considered at $95 \%$ confidence interval, and 2 - tailed p-values less than or equal to 0.05 were accepted to imply significant differences between sets of data compared.

\section{RESULTS}

\section{Age and sex distribution of patients}

The age and sex distribution of the 218 patients who fully participated in this study are given in Table 1 . This number excluded those who either defaulted or withdrew from the study on account of convenience. There was no significant difference between the number of males and females, and the number of children below 5 years when compared with other patients from 5 years and above ( $p$ $>0.05$ ).

\section{Clinical and parasitological responses to chloroquine}

The clinical and parasitological responses to chloroquine are shown in Table 2. Chloroquine produced a clinical success of $74.3 \%$ in the eradication of malaria parasites in the patients but parasitological success was only achieved in $45 \%$ of the patients. The proportion of patients with clinical failure or parasitological failure were significantly higher in patients from 5 to 48 years old than patients below 5 years $(p \leq 0.001)$. Furthermore, the proportion of patients from 5 to 48 years old with clinical failure was more than twice the proportion of patients below 5 years with clinical failure. Also, the proportion of patients from 5 to 48 years years old with parasitological failure was nearly doubled the proportion of patients below 5 years with parasitological failure. Gender had no significant effect on either the clinical response or parasitological response to chloroquine $(p>0.05)$.

\section{Level of resistance to chloroquine}

Class II (R2) and class III (R3) types of resistance were found to have occurred in $81(37.2 \%)$ and $38(17.4 \%)$ of the patients, respectively. We found no significant differences in the levels of resistance in males when compared to those of the females $(p>0.05)$. 
Table 2. Clinical and parasitological response of chloroquine in patients with $P$. falciparum malaria.

\begin{tabular}{|l|l|l|l|l|c|}
\hline \multirow{2}{*}{ Response } & \multicolumn{2}{|c|}{ Age (years) } & \multicolumn{2}{c|}{ Sex } & \multirow{2}{*}{ Total (\%) } \\
\cline { 2 - 5 } & $\begin{array}{l}<5(\%) \\
\mathrm{n}=78\end{array}$ & $\begin{array}{l}5-48(\%) \\
\mathrm{n}=140\end{array}$ & $\begin{array}{l}\text { Male } \\
\mathrm{n}=113\end{array}$ & $\begin{array}{l}\text { Female } \\
\mathrm{n}=105\end{array}$ & \\
\hline Clinical failure & $10(12.8)^{\mathrm{b}}$ & $46(32.9)^{\mathrm{b}}$ & $28(24.8)$ & $28(26.7)$ & $56(25.7)$ \\
Parasitological failure & $27(34.6)^{\mathrm{c}}$ & $93(66.4)^{\mathrm{c}}$ & $62(54.9)$ & $58(55.2)$ & $120(55.0)$ \\
\hline
\end{tabular}

${ }^{b} p=0.001 ;{ }^{c} p<0.001$

\section{DISCUSSION}

Drug resistance in malaria is the ability of the parasite strains to survive and/or multiply despite administration and absorption of a drug given in doses equal to or higher than those usually recommended but within the limits of tolerance of the subject (Bruce, 1986). The World Health Organization (WHO) categorized resistance in malaria as S (sensitive), R1, R2 and R3 based on in vivo test (Bruce, 1986). This test was replaced in 1996 by WHO with the Therapeutic Efficacy Test based on clinical and parasitological criteria - a pragmatic test for National Malaria Control Programme (WHO, 1996). We have used these two approaches to evaluate the effectiveness of chloroquine in the five communities studied. Our results have shown that chloroquine is still effective in the treatment of uncompicated $P$. falciparum malaria in the communities despite the high prevalence of chloroquineresistant malaria in the region. This is evident in the clinical success of chloroquine in $74.3 \%$ of the patients. However, parasitemia was still evident in $45 \%$ of the patients by the 7th day after the first dose of chloroquine was administered, indicating resistance to chloroquine. We recognise that a mixed population of malaria parasite strains (susceptible and resistant) can be present in the blood of the patients. In this situation, the susceptible strains would be killed by the chloroquine in the early part of the treatment, making way for more resistant and virulent strains to multiply later (Wernsdorfer et al., 1995).

Children under the age of 5 years are known to be the most susceptible group to malaria mortality (WHO, $2002 \mathrm{~b}$ ). The severity and prevalence of malaria are also higher in this age group than older children and adults (May et al., 1999; Mockenhaupt et al., 2000). Furthermore, at equal dosages per body weight, plasma chloroquine concentrations are lower in children below 5 years of age than older children and adults (Maitland et al., 1997). These indications would suggest that the therapeutic failure of chloroquine in malaria treatment is likely to be higher in children younger than 5 years than older people. Conversely, our results indicate that children younger than 5 years had much lower therapeutic failure rates than older people. The reason for this is unclear. Earlier report indicates that mixed species infections of malaria parasites are higher in children below 5 years (Mockenhaupt et al., 2000) than older people. Malaria infections with parasite strains other than $P$. falciparum have been suggested to act as natural vaccines preventing severe manifestations of $P$. falciparum infections (Williams et al., 1996). If this suggestion holds, clearance of $P$. falciparum malaria in the patients below 5 years of age could be enhanced, as none of them had severe malaria. Furthermore, long-term exposure to chloroquine, particularly by presumptive chloroquine treatment or prophylaxis, are prerequisites for the emergence of chloroquine resistance (Mockenhaupt et al., 2000). This situation is less likely to occur in children below the age of 5 years than older people. The efficacy of chloroquine is thus more likely to be lower in people older than 5 years than those below 5 years of age. Greater awareness on the need for early and proper treatment in chlidren below 5 years of age may also be a contributing factor. Gender appears not to play a role in therapeutic efficacy of chloroquine as we found no significant difference between the frequency of therapeutic failures in males as compared to females.

Increasing resistance of $P$. falciparum malaria to chloroquine has earlier been reported in some parts of Nigeria (Umotong et al., 1991). Survellance network in the country from mid-1987 to 1990 revealed that chloroquine resistant $P$. falciparum (CRPF) was widespread (Ekanem, 1997). In the northern part of the country, parasitological failures increased from $18.7 \%$ in 1988 to $24.5 \%$ in 1995 (Molta, 1995). From 1987 to 1997, resistance ranging from $33 \%$ to $72 \%$ was also reported in the southern part of the country (Ekanem, 1997). Although the parasitological failure found in our study is similar to the $53.6 \%$ found in Calabar, southern Nigeria in 1991 (Umotong et al., 1991), stability of resistance to chloroquine in the area studied cannot be inferred because the two areas (Calabar and Delta region) are in different locations. Clinical failure of $25.7 \%$ recorded in this study is also similar to the $22 \%$ reported in Ibadan, southern Nigeria in 1997 (Ekanem, 1997; Oduola, 1997). The frequencies of clinical and parasitological failures following chloroquine treatment are indicative of the continuous emergence of chloroquine resistance strains of $P$. falciparum in the communities studied and most likely in other parts of the country. These frequencies are at unacceptable levels having exceeded the $15-24 \%$ set by WHO (2002a) as action phase. Thus, the need for action to be taken to address the problem in the 
communities and other parts of the country has become very urgent.

Based on WHO earlier categorisation of resistance to antimalarial drugs (class I, R1; class II, R2 and class III, R3), our results have demonstrated high proportions of R2 $(37.2 \%)$ and R3 (17.4\%) resistance in the communities studied. The R3 resistance was similar to that reported in 1991 in Calabar (Umotong et al., 1991). Studies have shown that R3 resistance to chloroquine is not present in some areas in Congo (Carme et al., 1998) and Madagascar (Raharimalala et al., 1995) but present at a very low level in Coté d'Ivoire (Henry et al., 1998). R3 resistance in this study is lower than the $23 \%$ reported in Zimbabwe (Mohamva et al., 1996) and the $22 \%$ reported in Port Moresby, Papua New Guinea (Hombhanje, 1997). However, the R2 resistance in our results is much higher than the $10 \%$ reported in Zimbabwe (Mohamva et al., 1996) and the 4\% reported in Papua New Guinea (Hombhanje, 1997). High, rather than low, doses of chloroquine is believed to induce R3 resistance to chlroquine (Henry et al., 1998).

The mechanism of resistance to chloroquine in the communities studied is not clear, since the mechanism of resistance to antimalarial drugs is still debatable (Wellems et al., 1990; Rathod et al., 1997; Phillips, 2001). However, resistance to chloroquine has been reported to be due to reduced uptake of the drug by the infected erythrocyte and presumably reflects a reduction in the accumulation of the drug in the parasite's lysosome (Phillips, 2001; Warhurst, 2001). Association between chloroquine resistance and mutations in an mdr-like gene (pfmdr-1) has been reported (Warhurst, 2001). There is evidence to suggest that a mutation in pfcrt is required to confer a basic level of resistance before mutations in pfmdr-1 can have an effect; the basis for the suggested use of a rapid and sensitive test to detect pfort T76 in blood samples to ascertain the effectiveness of chloroquine (Warhurst, 2001; Reed et al., 2000). A number of factors have been identified as playing a role in the emergence of resistance to chloroquine. These include poor compliance to drug therapy, host immunity, use of sub-therapeutic doses, mutation and frequency of re-infection (Hombhanje, 1997; Phillips, 2001; Werndorfer, 1991).

Treatment of uncomplicated malaria in the Niger-Delta region (where this study was carried out) is frequently based on the fact that as long as chloroquine is effective enough, there is very little incentive for basing its use on reliable diagnostic confirmation. As in the communities studied, resistance to antimalarial drugs is proving to be a challenging problem in malaria control in most parts of the world. A fundamental aspect of drug usage recommendations is the impact of resistance on morbidity and mortality and hence the impact of the timing and nature of a policy decision on the health of the people in the community studied and the rest of the country. The principle of combination drug in malaria chemotherapy has been established as a measure to improve treatment efficacy, delay the emergence of drug resistance, and reduce the prevalence of gametocyte carriage which could, in turn, reduce transmission of malaria parasites (WHO, 2001a,b). Cost considerations in many parts of the country would support the use of empirical treatment with chloroquine or an alternative first-line drug, such as sulfadoxine-pyrimethamine (Warhurst, 2001), in patients with uncomplicated malaria. In the case of more expensive drug regimens, the cost would have to be balanced against the potential savings in the cost of treatment. Reliable, microscopy-based diagnosis should be mandatory in view of the financial implications when much more expensive alternative drugs need to be used when resistance to chloroquine occurs.

\section{Conclusion}

Chloroquine is still effective in the treatment of most cases of uncomplicated $P$. falciparum malaria in some communities in Delta State, Nigeria. However, in many cases of uncomplicated $P$. falciparum malaria, resistance to chloroquine is likely and intervention with alternative antimalarial drugs may be required for complete clearance of parasitemia in the patients. Despite the limited communities in which this study was carried out, our results may be used as an important indicator of the significant level of therapeutic failure of uncomplicated $P$. falciparum malaria to chloroquine in Nigeria. Additional drug efficacy assessment throughout the country is needed to support national antimalarial drug policy.

\section{ACKNOWLEDGEMENTS}

We are grateful to the staff and management of Central Hospital, Warri and Calvary Medical Laboratory for their support. This work was carried out with the financial support from the Arienmughare family of Warri, Delta State, Nigeria.

\section{REFERENCES}

British Pharmacopoeia (1998). The Pharmaceutical Press, London. Bruce CLJ (1986). Chemotherapy of Malaria. WHO Technical Report Series No. 27, Geneva, World Health Organization, Geneva.

Carme B, Ndounga M, Kissila AM, Samba G, Baya TN (1998). No variation in chloroquine resistance (Plasmodium falciparum) from 1986 to 1996 in semi-immune children in Brazzaville (Congo). Bull. Soc. Pathol. Exot. 91: 164-166.

Ejov MN, Tun T, Aung S, Sein K (1999). Response of falciparum malaria to different antimalarials in Myanmar. Bull. World Health Org. 77: 244-249

Ekanem OJ (1997). Use of antimalarial drugs in Nigeria. National symposium on malaria in Nigeria, Nigeria Institute for Medical Research, Lagos, Nigeria.

Henry M, Koné M, Guillet P, Mouchet J (1998). Resistance to chloroquine and malaria control in the Ivory Coast. Santé 8:287-291. 
Hombhanje FW (1997). Parasitological response of Plasmodium falciparum infection to chloroquine treatment in malaria patients in Port Moresby. PNG. Med. J. 40: 74-78.

Maitland K, Williams TN, Kotecka BM, Edstein MD, Rieckmann KH (1997). Plasma chloroquine concentrations in young and older malaria patients treated with chloroquine. Acta Trop. 66:155-161.

Malaria Foundation International (1998). Report on the multilateral initiative on malaria meeting on antimalarial drug usage and resistance. http://www.malaria.org/ppt/drug.html; assessed Sept 25, 2003.

May J, Mockenhaupt FP, Ademowo OG, Falusi AG, Olumese PE, Bienzle U, Meyer CG (1999). High rate of mixed and subpatent malarial infections in southwest Nigeria. Am. J. Trop. Med. Hyg. 61: 339-343.

Mockenhaupt FP, May J, Bergqvist Y, Ademowo OG, Olumese PE, Falusi AG, Gro terlinden L, Meyer CG, Bienzle U (2000). Concentrations of chloroquine and Malaria parasites in blood in Nigerian children. Antimicrob. Agents Chemother. 44: 835-839.

Mohamva Al, Peterson DE, Rakata L (1996). Chloroquine resistant falciparum malaria in Mutare District, eastern Zimbabue. Cent. Afr. J. Med. 42: 112-113.

Molta NB (1995). Susceptibility of Plasmodium falciparum to malarial drugs in North-eastern Nigeria. Trans. Roy. Trop. Med. Hyg. 89: 422-425.

Neequaye J, Coe-Ene J, Taelman H (1986). In vivo chloroquine resistant falciparum malaria in West Africa. Lancet 1: 153-154.

Oduola AMJ (1997). Susceptibility of $P$. falciparum to antimalarial drugs in vitro in Nigeria. National symposium on malaria in Nigeria, Nigeria Institute for Medical Research, Lagos, Nigeria.

Peters W (1998). Drug resistance in malaria parasites of animals and man. Adv. Parasitol. 41: 1-62.

Phillips RS (2001). Current status of malaria and potential for control. Clin. Microbiol. Rev. 14: 208-226.

Raharimalala L, Rabarison P, Pepers-Rason MD, Pepers JP, Ramambanirina L, Rason MA, Jambou R, Roux J (1995). Drug resistance and therapeutic strategy. Sante 5: 389-392.

Rathod PK, McErlean T, Pei-Chieh L (1997). Varaitions in frequencies of drug resistance in Plasmodium falciparum. Proc. Natl. Acad. Sci. USA. 94: 9389-9393.

Reed MB, Saliba KJ, Caruana SR, Kirk K, Cowman AF (2000). Pgh1 modulates sensitivity and resistance to multiple antimalarials in Plasmodium falciparum. Nature 403: 906-909.

Salako LA, Sowunmi A, Walker O (1990). Evaluation of the clinical efficacy and safety of halofantrin in falciparum malaria in Ibadan, Nigeria. Trans. Roy. Soc. Trop. Med. Hyg. 84: 644-647.
Sowunmi A, Salako LA, Walker O, Ogundahunsi OA (1990). Clinical efficacy of mefloquine in children suffering from CRPF in Nigeria. Trans. Roy Soc. Trop. Med. Hyg. 84: 761-764.

Sowunmi A, Salako LA (1992). Evaluation of the relative efficacy of various antimalarial drugs in Nigerian children under 5 years of age suffering from acute uncomplicated falciparum malaria. Ann. Trop. Med. Parasitol. 86: 1-8.

Umotong $A B$, Ezedinachi EN, Okerengwo AA, Usanga EA, Udo JJ, Williams Al (1991). Correlation between in vivo and in vitro response of choloroquine resistant Plasmodium falciparum in Calabar, SouthEastern Nigeria. Acta Tropica. 49: 119-125.

Wellems TE, Panton LJ, Gluzman IY, do Rosario VE, Gwardz RW, Walker-Jonah A, Krogstad DJ (1990). Chloroquine resistance not linked to mdr-like genes in Plasmodium falciparum. Nature 345: $202-$ 203.

Wernsdorfer WH (1991). The development and spread of drug resistant malaria. Parasitol. Today 7: 297-303.

Wharhurst DC (2001). A molecular marker for chloroquine-resistant falciparum malaria (editorial). New Engl. J. Med. 344: 299-302.

Williams TN, Maitland K, Bennett S, Ganczakonski M, Peto TE, Newbold Cl, Bowden DK, Weatherall DJ, Clegg JB (1996). High incidence of malaria in alpha-thalassaemic children. Nature. 383: 522-525

World Health Organisation (1992). WHO/DAP/92.3, How to investigate drug use in communities. World Health Organization, Geneva, Switzerland

World Health Organisation (1996). WHO/MAL/96.1077, Assessment of therapeutic efficacy of antimalarial drugs for uncomplicated falciparum malaria in areas of intense transmission. World Health Organization, Geneva, Switzerland.

World Health Organisation (2001a). WHO/CDS/RBM/2002.33, Use of antimalarial drugs. Report of a WHO informal consultation. World Health Organisation, Geneva, Switzerland.

World Health Organisation (2001b). WHO/CDS/RBM/2002.35, Antimalarial drug combination therapy. Report of a WHO technical consultation. World Health Organisation, Geneva, Switzerland.

World Health Organisation (2002a). Antimalarial drug resistance: Guidelines for surveillance and containment. World Health Organisation, Geneva, Switzerland.

World Health Organisation (2002b). WHO/CDS/RBM/2002.42, Community involvement in rolling back malaria. World Health Organisation, Geneva, Switzerland. 